\title{
Linx
}

Revue des linguistes de l'université Paris X Nanterre

66-67| 2012

Dynamiques de la construction des sens attendus et inattendus dans les langues

\section{De l'in/attendu dans les énoncés concessifs avec MAY}

Illustration à partir d'un discours de Barack Obama

\section{Stéphane Gresset}

\section{OpenEdition \\ Journals}

\section{Édition électronique}

URL : http://journals.openedition.org/linx/1446

DOI : $10.4000 /$ linx. 1446

ISSN : 2118-9692

\section{Éditeur}

Presses universitaires de Paris Nanterre

Édition imprimée

Date de publication : 15 septembre 2012

Pagination : $51-67$

ISSN : 0246-8743

Référence électronique

Stéphane Gresset, "De l'in/attendu dans les énoncés concessifs avec MAY », Linx [En ligne], 66-67 | 2012, mis en ligne le 15 septembre 2013, consulté le 01 mai 2019. URL : http://

journals.openedition.org/linx/1446; DOI : 10.4000/linx.1446 


\title{
De l'in/attendu dans les énoncés concessifs avec MAY Illustration à partir d'un discours de Barack Obama
}

\author{
Stéphane Gresset (CLILLAC-ARP EA 3967) \\ Université Paris Diderot
}

\section{Préambule}

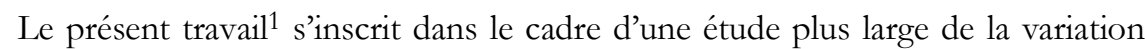
dans l'expression de la concession en anglais contemporain au sein d'extraits de corpus spécifiques, l'objectif étant d'apporter des éléments de réponse à la question de la justification du recours à tel marqueur ou schéma concessif dans tel contexte ; autrement dit, peut-on mettre au jour des indices contextuels (au sens restreint de cotextuels) et/ou discursifs (au sens large) susceptibles d'ouvrir la voie à, autrement dit de frayer ${ }^{2}$ l'apparition d'un schéma concessif plutôt qu'un autre et, toute différence de forme étant significative, de quelle variation cette différence est-elle révélatrice ?

Le propos de cette première contribution est d'illustrer la démarche en examinant, à partir d'un unique document, un discours d'Obama d'à peine 5000 mots,

\footnotetext{
${ }^{1}$ Mes très chaleureux remerciements à Ronald Flintham pour les discussions extrêmement fructueuses que j'ai pu avoir avec lui. Cet article lui doit bien plus qu'il ne le croit.

${ }^{2}$ Le concept de «frayage», qui renvoie aux conditions d'apparition ou encore aux énoncés précurseurs de tel ou tel marqueur, est emprunté à Culioli (1999, p. 135 et 1990, p. 124).
} 
les conditions d'apparition en contexte de plusieurs tours concessifs dont le point commun est de comporter une occurrence du modal MAY. Plus exactement, les quelques schémas concessifs de ce discours contenant tous MAY, nous en avons fait le point de départ de notre étude, mais sans pour autant (1) que nous postulions l'existence d'un «MAY concessif » (d'où notre formulation «avec MAY » et non « en MAY»), et (2) que nous nous intéressions exclusivement à MAY. Une fois en effet que nous aurons rappelé le rôle que joue ce modal dans ce type d'emploi, notre intérêt se portera tout autant sur les marqueurs associés à MAY dans ces schémas concessifs, en l'occurrence BUT, AS et WHILE, ainsi qu'à titre de comparaison et de manière ici plus allusive sur AL/THOUGH, YET, HOWEVER, EVEN IF notamment. Mais il nous faut, au préalable, rappeler ce que recouvre généralement la notion de concession, ne serait-ce que parce que nous serons amenés à en interroger certaines des propriétés caractéristiques.

\section{Les termes du débat}

Nous partirons de deux traits définitoires de la concession qui, tout en s'inscrivant dans le cadre de la théorie des opérations prédicatives et énonciatives d'Antoine Culioli, font l'objet d'un large consensus : « tout énoncé concessif repose sur la déconstruction d'une relation inférentielle normale entre contenus propositionnels.» (Ranger, 1998, p. 32), et la concession, ajoute Charreyre, « relève d'une relation primitive à l'intérieur d'une même socio-culture» (2007, p. 164, note 3). On retrouve la forme schématique ou abstraite de la concession dont on peut, à partir de Culioli (1999, p. 179), proposer la formulation suivante : là où $\mathrm{p}$ (la valeur du domaine notionnel associé à la première relation prédicative) entraîne normalement $\mathrm{q}$ (pour la seconde relation prédicative), et où $\mathrm{p}^{\prime}$ (autre que $\mathrm{p}$ ) entraîne normalement q' (autre que q), on est, dans un schéma à interprétation concessive, en présence à la fois de p' et de $\mathrm{q}$, c'est-à-dire de deux valeurs entre lesquelles le lien est problématique, autrement dit inattendu, là où les termes de «norme » et de « relation primitive » renvoient à ce qui est attendu.

Par ailleurs, la formulation de «déconstruction d'une relation inférentielle normale » pose la question centrale ici de la norme. Cette norme est-elle préconstruite en tant qu'elle relève de relations primitives fondées sur des propriétés physico-socioculturelles $^{3}$, ou bien est-elle le résultat de la construction en discours de relations entre notions ? Cette interrogation rejoint la réflexion de Sekali (1992, p. 113) sur l'implicite qu'elle considère, dans le cadre de son étude sur BUT, comme un construit, «un produit de la structuration linguistique », pas nécessairement un présupposé culturel.

\footnotetext{
${ }^{3}$ De Vogüé (1992, p. 25) fait, selon Ranger (1998, p. 28-29), « [un] rapprochement entre les relations primitives qui relient les notions et l'inférence qui sous-tend tout énoncé concessif : 'll'exemple Il est malheureux bien que riche.] s'interprète sans difficulté : il est fait état d'une corrélation usuelle (...) [qui] met en jeu une relation de cause à effet entre la richesse et le bonheur, que l'on ne cautionne pas nécessairement, mais que l'on restitue spontanément pour interpréter (l'exemple) : c'est ce type de relation que Culioli appelle relation primitive, dont il dit qu'elle se fonde sur des propriétés physicoculturelles.' »
} 
De ce point de vue, le terme d'« inférence discursive » nous parait plus satisfaisant que celui de "norme » mais également suffisant en ce qu'il permet de rendre compte aussi bien de la recevabilité d'exemples canoniques tels que «He may be a cop, but he isn't a bastard.» ou "The sun may rise the East, but it sets in the West. », que de celle de cas plus atypiques mais tout aussi acceptables en contexte approprié tels que «He may be a cop, but he is a bastard.» ou encore "He may have been to Yale, but he's intelligent». Aucune raison en effet de rejeter ce dernier, même en l'absence d'occurrence attestée; il suffirait de créer en contexte les conditions nécessaires, plutôt inattendues certes, pour que cette séquence devienne un énoncé, en préconstruisant une inférence selon laquelle, par exemple, l'argent nécessaire pour être admis à Yale pourrait ne pas être considéré par tous comme une preuve d'intelligence ; la relation entre les notions GO TO YALE et BE INTELLIGENT serait du coup considérée comme n'allant pas de soi, autorisant de ce fait l'énoncé concessif proposé.

De cette première interrogation, sur la notion même de concession, en découle une seconde, terminologique mais aussi théorique, à propos du tour ou schéma concessif qui nous occupera en premier lieu, à savoir la corrélation... MAY... BUT... Plutôt que de "structure concessive », nous parlerons en effet de "schéma concessif » (bien que même cela nous paraisse un raccourci discutable). La corrélation... MAY... BUT... est simplement un moyen parmi d'autres d'exprimer la démarche concessive que nous avons explicitée ci-dessus; c'est un schéma contribuant à l'expression de la concession dont il est question. Incontestablement, et pour tout autre marqueur que THOUGH et ses «composés » (ALTHOUGH, EVEN THOUGH), le mouvement concessif relève davantage de la pragmatique que de la linguistique au sens strict de renvoi à des marqueurs spécifiques. Concéder, n'est-ce pas, comme le dit Claude Charreyre $^{4}$, «entrer dans le jeu de l'autre sans pour autant considérer que les arguments avancés (par l'autre dans le dialogue, par le co-énonciateur dans la construction de sa propre argumentation en tant qu'énonciateur ${ }^{5}$ ) sont les seuls possibles. »?

Or cette dimension essentielle de la concession qu'est l'intersubjectivité ${ }^{\text {n'est }}$ observable et analysable qu'en contexte et plus précisément dans du corpus continu, pour que l'on puisse d'une part suivre le raisonnement et l'argumentation de bout en bout, d'autre part comparer les différentes occurrences du même marqueur ou de la même corrélation de marqueurs, enfin et surtout, car c'est l'enjeu que nous nous sommes fixés, comparer les diverses manifestations ou expressions de la concession. C'est pourquoi nous avons opté pour un corpus restreint mais intégral et donc homogène, plutôt que pour des occurrences aussi variées que possible issues de corpus électroniques tels que le BNC ou le COCA mais du coup tirées de sources différentes.

\footnotetext{
${ }^{4}$ Communication personnelle.

${ }^{5}$ Le co-énonciateur comme émanation de l'énonciateur, comme la représentation qu'il construit de l'autre dans le discours. (Sekali, 2007, p. 157)

${ }^{6}$ À noter que cela rejoint, dans un tout autre cadre, la présentation d'Eve Sweetser d'un emploi métalinguistique ou pragmatique de MAY dans le cas de la concession (Sweetser, 1990, p. 69-75).
} 
Stéphane Gresset

Corpus : Obama's Philadelphia Speech on Race and the Race, «A More Perfect Union»

Alors en campagne pour l'investiture démocrate, le Sénateur Obama prononça le discours en question en mars 2008, au beau milieu d'une polémique dont il perçut vite qu'elle pourrait lui coûter cher. Obama répond en effet ici à de sévères critiques émises à son encontre suite au soutien très controversé qu'il reçut de son ancien pasteur de Chicago, le Révérend Wright, qui avait tenu, peu de temps auparavant, des propos très provocateurs. Sur le fond donc, Obama est pris entre deux pressions, se désolidariser des propos du Révérend, voire les condamner, mais sans pour autant désavouer complètement l'homme; dans cette opposition même apparaît déjà l'intérêt de la démarche concessive, tout particulièrement dans sa dimension intersubjective concéder à l'autre sans pour autant se renier soi-même, autrement dit céder mais ne céder que partiellement, et surtout ne pas se désavouer. Sur la forme maintenant, ce discours ${ }^{7}$ nous a paru particulièrement pertinent dans la mesure où, quoique relativement bref (environ 5000 mots), il contient 10 occurrences du modal MAY dont 6 dans des segments à interprétation nettement concessive, associé à BUT pour 4 d'entre elles, à WHILE et à ... AS... AS... dans un cas chacun. Aucune occurrence, en revanche, de THOUGH ou d'une forme apparentée (ALTHOUGH ou EVEN THOUGH), ni de EVEN IF ou de IF seul, subordonnants qui peuvent aussi, dans cette même interprétation, être accompagnés de MAY. Autrement dit, dans ce discours éminemment concessif dans l'esprit, parce qu'Obama n'a pas le choix et que c'est la raison d'être de son intervention, il n'use, pour exprimer la concession, que de certains des moyens dont dispose la langue, ce qui nous donnera l'occasion, in fine, d'esquisser une hypothèse sur la distribution spécifique des moyens ici mis en œuvre.

L'analyse qui suit des énoncés concessifs de ce discours a donc pour objectif de répondre à deux séries de questions, la première d'ordre onomasiologique et la seconde sémasiologique :

- en quoi consiste précisément en contexte la démarche concessive, autrement dit quelles conditions doivent être réunies pour qu'on puisse en parler sans ambiguité et à quoi correspond dans chaque exemple l'inférence préconstruite dont on a postulé l'existence?

- pourquoi ne trouve-t-on, dans ce discours à la tonalité concessive par endroits manifeste, aucune occurrence d'un des subordonnants concessifs classiques tels que THOUGH, ALTHOUGH, EVEN THOUGH ou EVEN IF, et qu'en serait-il s'ils étaient utilisés à la place des marqueurs effectivement utilisés - avec les questions qui s'ensuivent pour toute manipulation, s'ils sont possibles quelle différence, et s'ils ne le sont pas pourquoi pas?

\footnotetext{
${ }^{7}$ Un grand merci à notre collègue Laure Lansari pour avoir la première attiré notre attention sur ce discours, que l'on peut écouter et dont on trouvera la transcription intégrale sur le site suivant, parmi d'autres : http://www.huffingtonpost.com/2008/03/18/obama-race-speech-read-th_n_92077.html
} 


\section{Le traitement des énoncés}

\section{1. ...MAY .., BUT ...}

Exemple 1: I chose to run for the presidency at this moment in history because I believe deeply that we cannot solve the challenges of our time unless we solve them together - unless we perfect our union by understanding that we may have different stories, but we hold common hopes; that we may not look the same and we may not have come from the same place, but we all want to move in the same direction - towards a better future for our children and our grandchildren.

Ce premier énoncé constitue un bon point de départ pour illustrer la démarche concessive car le contexte est très explicite : évidences partagées par tous, we have different stories / we don't look the same / we don't come from the same place constituent des assertions sous-jacentes et MAY ne peut en aucun cas recevoir une interprétation épistémique ; on peut par ailleurs très facilement reconstituer les inférences :

'we - have different stories ${ }^{\prime}=>$ 'we - hold different hopes'

'we - not look the same/we - not come from the same place' => 'we - not move in the same direction'

Or ces inférences, dans ce cas précis, ne fonctionnent pas; à not the same story/look/origin est associé same hopes/direction, introduisant ainsi un hiatus par rapport à l'inférence préconstruite. Quel est le rôle de MAY dans cette interprétation?

Exemple 1a: ...- unless we perfect our union by understanding that we have different stories, but we hold common hopes; that we do not look the same and we do not come from the same place, but we all want to move in the same direction - towards a better future for of children and our grandchildren

Les deux variantes, avec ou sans MAY, ne sont pas équivalentes. En l'absence de MAY, les assertions que nous avons considérées comme sous-jacentes sont désormais explicites et ne donnent lieu à aucun jeu : la valeur assertée est la seule présente et il n'y a donc aucune altérité sur la première relation prédicative. Disparait du coup la dimension rhétorique et fortement intersubjective de l'extrait, c'est-à-dire la prise en compte du point de vue de cet autre auquel est attribuée la prise en charge de l'assertion sous-jacente mentionnée.

Par ailleurs, à côté de ... MAY... BUT..., AL/THOUGH (sans MAY et en conservant l'ordre des propositions c'est-à-dire avec antéposition de la subordonnée) parait tout à fait recevable :

Exemple 1b: ... that although we have different stories, we hold common hopes; that although we do not look the same and we do not come from

${ }^{8}$ Les apostrophes simples ( $(. .$.$) ) renvoient à la valeur (positive ou négative selon le cas) du domaine$ notionnel associé à la relation prédicative considérée. 
Stéphane Gresset

the same place, we all want to move in the same direction - towards a better future for of children and our grandchildren

Dans un cas comme dans l'autre, corrélation en ... MAY... BUT... ou subordination en ALTHOUGH, on a affaire à une structure en deux temps, avec deux options. Soit, comme c'est le cas en (1b) avec AL/THOUGH sans MAY, la valeur 'we - have different stories' est préconstruite comme validée et l'altérité, autrement dit 'we - not have different stories' est explicitement écartée ; on est alors dans un cas de « oui, et rien d'autre ». Soit, comme dans le contexte d'origine en (1) et même si 'we - bave different stories' est le cas en situation, l'altérité est prise en compte et on est alors dans un cas de « oui, entre autres ». Une simple coordination adversative avec BUT tel qu'en (1a), qui pourrait correspondre à un cas de « oui, sans plus », paraît insuffisante car la première relation sert ici de point de départ, de repère à une inférence qui, sans AL/THOUGH ou MAY (voire les deux), ne peut au mieux être qu'implicite ce qui, dans ce contexte, ne suffit pas à la mise en relief de la seconde proposition si caractéristique de la démarche concessive?

Avec AL/THOUGH la valeur de la relation prédicative associée ('we - have different stories', 'we - not look the same' ...) est explicitement concédée par l'énonciateur au co-énonciateur et de ce fait non remise en question, là où avec MAY (associé à BUT) cette valeur, tout à fait susceptible d'être validée, n'est présentée que comme l'une des valeurs possibles du fait de la présence, avec MAY, d'une altérité de fondation - d'où ces explicitations en français par « que nous ayons ou pas ... peu importe ...» ou bien « nous avons beau avoir ... ». L'énonciateur, dans ce contexte précis, cède bien moins au co-énonciateur qu'avec AL/THOUGH puisque, tout en prenant en compte la position de l'autre, il met sur le même plan les deux valeurs du domaine associé à la relation. De ce point de vue, Obama n'aurait-il pas davantage recours à ... MAY... BUT... qu'à AL/THOUGH dans l'expression de la concession parce que reconnaitre avec AL/THOUGH la position de l'autre comme acquise car validée, ce serait se mettre dans une position de plus grande faiblesse, là où avec ... MAY... BUT... il sauve plus efficacement la face ?10 Toujours est-il que la concession, nous semble-t-il, doit être pensée en termes de degrés, le statut accordé à la valeur dite " concédée » étant variable ${ }^{11}$; mais c'est là une hypothèse qui exige d'être étayée.

Troisième et ultime manipulation ; après avoir comparé MAY et AL/THOUGH, opposons BUT à YET :

Exemple 1c: ... unless we perfect our union by understanding that we may have different stories, yet we hold common hopes; that we may not look

\footnotetext{
${ }^{9}$ Reste le cas, si l'on se réfère au carré logique de Culioli, de «oui, en tout cas », qui pourrait correspondre à l'association de ALTHOUGH avec MAY, mais cela dépasse le cadre du présent article.

${ }^{10}$ De ce point de vue, la locution française «Au temps/Autant pour moi » est plus proche de la démarche marquée par ... MAY... BUT... que de ALTHOUGH.

${ }^{11}$ Huddleston \& Pullum (2002, p. 735) intitulent ainsi une sous-partie de leur exposé : «Variation in the strength of the contrary-to-expectation implicature ».
} 
the same and we may not come from the same place, yet we all want to move in the same direction - towards a better future for of children and our grandchildren

Alors que le coordonnant BUT marque explicitement l'adversativité ${ }^{12}$, donc un changement d'orientation avec, dans le cas qui nous occupe, lecture rétrospective du MAY de la première proposition et remise en cause de l'inférence préconstruite, nous ferons l'hypothèse qu'avec YET on a affaire à un contraste d'une autre nature, qui résulte de la combinaison des deux composantes que nous postulerons pour YET, à savoir la concomitance et la visée, en conformité avec son étymologie : till now ou moreover + now ; mixte de valeurs que l'on retrouve dans les autres emplois de YET, aussi bien dans «a part of our union that we have yet to perfect » (extrait du même discours) que dans son emploi aspectuel, associé à NOT, dans la variante du précédent « a part of our union that we haven't yet perfected». En (1c) «we may have different stories » et «we hold common hopes» coexistent au sens où ces deux contenus propositionnels sont concomitants, mais «till now », qui sous-entend une association inhabituelle ou inattendue ; le contraste, du coup, est implicite ou indirect, donc moins marqué qu'avec BUT, ce qui rend probablement compte de l'absence de ... MAY... YET ... dans les tours dits concessifs.

Notons que cette valeur mixte de concomitance et de visée vaut tout aussi bien pour les deux autres emplois de YET mentionnés : (1) « have yet to perfect» = « have to perfect till now» : au présent en tout cas, visée de 'we - perfect'; (2) « haven't yet perfected » = «not perfected till now»: ici, c'est l'état négatif 'not perfected' qui est le cas ... pour le moment en tout état de cause.

S'il nous est impossible d'en traiter ici, nous devrons cependant, dans une étude ultérieure, nous poser d'autres questions sur et autour de YET. Quelle est l'incidence, en contexte, de la présence ou non de MAY dans un énoncé avec YET ? YET est combinable avec des coordonnants : le discours d'Obama compte 3 exemples de « and + yet » et la grammaire de Quirk \& al. en propose un de « but + yet ». Enfin, YET doit être comparé avec STILL, marqueur avec lequel c'est du prolongement de l'état actuel (avec sa traduction par «il reste/demeure que ») qu'on déduit que le changement d'état est repoussé, STILL qui par ailleurs est également compatible avec AND.

Nous terminerons cette partie sur l'association entre MAY et BUT par quelques remarques sur l'énoncé (2), proche de (1) en apparence mais différent en réalité, et pas seulement parce qu'on a, avant BUT, un point et non une virgule.

Exemple 2: For the men and women of Reverend Wright's generation, the memories of humiliation and doubt and fear have not gone away; nor has the anger and the bitterness of those years. That anger may not get expressed in public, in front of white co-workers or white friends. But it does find voice in the barbershop or around the kitchen table. At times, that anger is exploited by politicians, to gin

\footnotetext{
12 L'étymologie de BUT est bien connue, «be-utan » signifiant « on the outside of, outside, without». BUT marqueur « d'une rupture par rapport à un autrui » (Sekali, 1992, p. 115).
} 
Stéphane Gresset

up votes along racial lines, or to make up for a politician's own failings. And occasionally it finds voice in the church on Sunday morning, in the pulpit and in the pews. The fact that so many people are surprised to hear that anger in some of Reverend Wright's sermons simply reminds us of the old truism that the most segregated hour in American life occurs on Sunday morning. That anger is not always productive; indeed, all too often it distracts attention from solving real problems; it keeps us from squarely facing our own complicity in our condition, and prevents the African-American community from forging the alliances it needs to bring about real change. But the anger is real; it is powerful; and to simply wish it away, to condemn it without understanding its roots, only serves to widen the chasm of misunderstanding that exists between the races. In fact, a similar anger exists within segments of the white community.

Ce point n'est en effet pas la raison mais plutôt la conséquence d'une différence avec le cas précédent, comme on le voit à l'examen des relations prédicatives en présence. Il n'y a pas, dans ce contexte, d'inférence entre 'anger - not get expressed in public' et 'anger - not find voice in the barbershop or around the kitchen table'. On ne peut, à propos de la colère dont il est très abondamment question dans l'extrait (12 références en une vingtaine de lignes), inférer 'not find voice in the barbershop or around the kitchen table' ...' de 'not get expressed in public'; il n'y a pas de lien nécessaire de cause à conséquence entre la sphère publique de la première proposition et celle du privé qu'évoquent «barbershop» et «kitchen table». Il y a certes une opposition entre le public et le privé, renforcée par la présence de l'auxiliaire DO, mais pas de concession à proprement parler ni la moindre relation intersubjective avec attribution de la valeur en question à une autre instance que l'énonciateur (coénonciateur ou interlocuteur). MAY reçoit ici une interprétation épistémique (parmi d'autres, cette éventualité-là n'est pas à exclure), il n'y a pas de retour sur son interprétation à la lecture de la proposition introduite par BUT et aucune relation inter-sujets par laquelle l'énonciateur se mettrait «à distance » de cette valeur sans pour autant la rejeter. On a là autant de conséquences de l'absence de relation inférentielle, et sans «attente » pas de «denial of expectation ». Enfin, mais cela nous entraînerait trop loin, il en serait autrement avec THOUGH, ALTHOUGH ou EVEN THOUGH, marqueurs explicitement concessifs qui préconstruiraient par définition une relation d'inférence qui n'est tout simplement pas présente dans l'environnement contextuel de cet exemple ; il n'y a pas ici, autrement dit, de frayage concessif.

\subsection{MAY en subordonnée}

Dans cette $2^{\text {ème }}$ partie, et toujours dans le cadre général de la variation dans l'expression de la concession, nous aborderons deux énoncés à interprétation concessive incontestable et comprenant un MAY mais cette fois dans une subordonnée, introduite dans un cas par WHILE et dans l'autre par la structure corrélative ... AS... AS... 
Exemple 3: For the African-American community, that path means embracing the burdens of our past without becoming victims of our past. It means continuing to insist on a full measure of justice in every aspect of American life. But it also means binding our particular grievances - for better health care, and better schools, and better jobs - to the larger aspirations of all Americans - the white woman struggling to break the glass ceiling, the white man who has been laid off, the immigrant trying to feed his family. And it means taking full responsibility for our own lives - by demanding more from our fathers, and spending more time with our children, and reading to them, and teaching them that while they may face challenges and discrimination in their own lives, they must never succumb to despair or cynicism; they must always believe that they can write their own destiny. Ironically, this quintessentially American - and yes, conservative - notion of self-help found frequent expression in Reverend Wright's sermons.

Le contexte laisse entrevoir, ou tout au moins n'est pas incomptabible avec une proposition telle que " our [i.e. American] children will face challenges and discrimination in their own lives», et l'on ne peut se contenter de dire qu'une interprétation épistémique de MAY est défendable parce que "while it is possible that they will face... " serait une glose recevable. Ce à quoi renvoie MAY est ici sa valeur de base, autrement dit un jeu sur les deux valeurs associées au domaine notionnel, ou encore, en termes culioliens, le passage de la valeur positive ou I comme intérieur du domaine 'they - face challenges and discrimination' à IE ou position décrochée 'they - face...'/ 'they - not face...'.

La subordonnée antéposée introduite par WHILE $^{13}$ sert, quant à elle, à introduire le repère de l'énoncé, par rapport auquel est repérée la principale, qui constitue le propos central de l'énoncé, pour ainsi dire «mis en attente» du fait de WHILE; WHILE suspend, fait patienter «le temps que...», pour renvoyer à son étymologie. Les deux relations prédicatives en présence sont construites comme concomitantes mais cette concomitance se situe au niveau des composantes qualitatives des occurrences de ces relations prédicatives, et non sur le plan quantitatif comme ce serait le cas dans l'interprétation temporelle de simultanéité de WHILE ${ }^{14}$. L'interprétation concessive de l'énoncé tient à ce que les relations ainsi construites comme concomitantes sont, pour ce qui est des relations primitives en jeu, notionnellement contradictoires. Dissymétrie syntaxique du fait de la subordination et de la nécessaire antéposition, et concomitance sémantique de deux relations prédicatives notionnellement contradictoires, telle est selon nous l'équation de la concession en WHILE.

On notera qu'il est ici, comme avec YET, question de concomitance et, outre qu'il serait intéressant de comparer des exemples en contexte de ces différents

${ }^{13}$ L'antéposition, sans être suffisante, est néanmoins nécessaire pour qu'une subordonnée en WHILE reçoive une interprétation concessive.

${ }^{14}$ Nous examinerons, dans le cadre d'une étude ultérieure et à partir des hypothèses de Chuquet \& Oriez 2000, la possibilité de la coexistence des deux paramètres Qlt et Qnt avec mise en relief de l'un ou de l'autre, ainsi par exemple Qlt (Qnt). 
marqueurs de la concomitance (... MAY..., YET... vs WHILE... MAY par exemple), on sera tenté de définir la concession, par opposition à la causalité selon Culioli (1990, p. 139), comme un mixte de concomitance et de non-consécution - de la concession donc comme une forme d'anti-causalité.

Autre exemple de subordination + MAY à interprétation concessive, l'énoncé (4), en ... AS... AS... MAY..., à partir d'une structure qui nous ramène précisément, mais par un autre biais, à ce mixte de concomitance et de non-consécution :

Exemple 4: The church contains in full the kindness and cruelty, the fierce intelligence and the shocking ignorance, the struggles and successes, the love and yes, the bitterness and bias that make up the black experience in America. And this helps explain, perhaps, my relationship with Reverend Wright. As imperfect as he may be, he has been like family to me. He strengthened my faith, officiated my wedding, and baptized my children. Not once in my conversations with him have I heard him talk about any ethnic group in derogatory terms, or treat whites with whom he interacted with anything but courtesy and respect. He contains within him the contradictions - the good and the bad - of the community that he has served diligently for so many years.

Le contexte est ici aussi particulièrement explicite : ont été évoquées juste avant l'extrait quelques-unes des critiques adressées au révérend, donc de son «imperfection» ("... incendiary language... widen the racial divid... denigrate... offend white and black alike. I have already condemned... the statements...»), et sont explicités par la suite des signes de ce que " he is like family to me " (He strengthened my faith, officiated my wedding, and baptized my children.» notamment). Enfin l'inférence déconstruite est très claire: "if he is (so) imperfect he shouldn't be like family to me ».

Avec toujours le même double objectif, d'une part de préciser les conditions d'emploi du ... MAY... BUT... concessif, et d'autre part de comparer ... MAY... BUT... à d'autres schémas concessifs, nous aborderons deux questions : quelle est la spécificité de la structure de l'exemple, autrement dit quels sont les rôles respectifs de la corrélation ... AS... AS... et de MAY, et en quoi cette structure se distingue-t-elle de ... MAY... BUT... ("He may be imperfect, but he has been like family to me. »)?

Commençons par décomposer la structure de l'exemple. On sait, avec les travaux de Flucha notamment, que AS est un marqueur d'identification qui, schématiquement, sert à identifier le degré associé au terme qui est à sa gauche au degré de celui introduit par AS. AS fonctionne comme un curseur qui fait se déplacer le terme de gauche par rapport au terme qui est à sa droite ou terme repère. Nous ne développerons pas ici la raison de la présence des deux AS sinon pour reprendre l'hypothèse émise par Charreyre (2005) et dire qu'avec "Imperfect as he may be, ...», et plus clairement encore avec "Imperfect as he is... », le degré d'imperfection ${ }^{15}$ attribué au révérend Wright est considéré

15 On rappellera que ce discours a pour titre « A More Perfect Union». 
comme établi, étalonné, mesuré, à partir des déclarations qui ont été les siennes, et de ce fait non susceptible d'être remis en question : « he is imperfect, and as such...» ou bien «Avec le degré d'imperfection qui est le sien et reconnu comme tel... ».

Avec la corrélation ... AS... AS..., on est en présence d'une double relation d'identification, établie en contexte et non pas au préalable donc, et sans étalonnage : «avec le degré d'imperfection qui est celui qui est le sien (quel qu'il soit donc), ... ». Il reste que, si la concession est compatible avec un seul AS comme avec deux, comment cette interprétation se construit-elle ? Une fois de plus, de la confrontation, c'est-à-dire de la mise en présence de deux assertions construites ici comme incompatibles car contradictoires : 'be is imperfect' / 'he is like family to me'. S'établit du coup entre ces deux propositions une relation qui ne peut être de simple simultanéité, leur coexistence étant problématique, et encore moins une relation de causalité puisque c'est précisément l'inverse qui se produit, et l'on parlera donc d'anti-causalité ('be imperfect' étant associé à l'opposé de ce qu'il est censé entraîner), dont la concession est une manifestation.

Avec ... MAY... BUT... ("He may be imperfect, but he has been like family to me.»), on passe de l'intérieur ou I à une position décrochée IE qui permet d'aller en I ou en $\mathrm{E}$, on part donc de l'intérieur du domaine (ce qui est clairement le cas ici avec le contexte amont, le point de départ du raisonnement étant incontestablement 'he is imperfect'), mais, du fait de MAY, I est présenté comme un possible parmi d'autres et l'altérité est réintroduite, autrement dit l'extérieur; or, l'intérieur étant mis au compte de l'autre, l'énonciateur n'écarte pas, implicitement au moins, que pour lui c'est l'extérieur qui prévaut ou prévaudrait - d'où ce jeu dont nous avons déjà parlé à propos de (1) ainsi que ces effets dialogiques si caractéristiques que permet ... MAY... BUT..., effets et jeu qui ne sont pas pertinents dans le cas qui nous occupe ; en effet, nul besoin ici de l'extérieur, précisément parce que le contexte amont l'exclut.

Avec ... AS... AS... + MAY, en effet, AS joue le rôle d'un curseur qui envisage tous les degrés possibles de la notion IMPERFECTION, qui en parcourt autrement dit toutes les quantités envisageables, tant qu'elles sont identifiées par le biais de la double opération à laquelle renvoie la corrélation ... AS... AS... que nous avons décrite plus haut. Le parcours que permet ce curseur s'effectue exclusivement sur l'intérieur du domaine, et à l'exclusion du degré $\varnothing$, c'est-à-dire de la quantité nulle, qui nous ramènerait à l'extérieur - un extérieur qui, rappelons-le, est pris en compte par le MAY de ... MAY... BUT.... Là où les choses se compliquent, c'est que MAY est présent dans (4) (... AS... AS... MAY...) comme dans la variante avec BUT (... MAY... BUT ...) et qu'il faut donc en rendre compte dans les deux cas ; c'est sa portée qui distingue ces deux emplois ou plutôt la nature de l'extérieur, autrement dit de l'altérité. Là où avec BUT, MAY sert à réintroduire l'extérieur du domaine, à savoir 'not be imperfect', par le biais d'un retour en position décrochée, avec ... AS... AS..., l'extérieur auquel renvoie MAY est constitué des différents degrés d'imperfection les uns par rapport aux autres.

Par ailleurs, qui dit parcours et concession pensera également à HOWEVER, d'autant que "However imperfect he may be, he has been like family to me.» est tout à fait 
recevable. Quelle différence alors avec la version d'origine ? Le parcours marqué par EVER dans son association à HOW parait cette fois être de nature non pas quantitative mais qualitative: "quelle que soit la nature, autrement dit le type d'imperfection qu'on puisse lui attribuer, ...» Parcours qualitatif donc mais qui, comme en (4), ne s'effectue que sur l'intérieur du domaine notionnel malgré la présence de MAY, davantage souhaitable dans la variante en HOWEVER que dans l'originale en ... AS... AS...; il nous semble en effet que « However imperfect he is, he has been like family to me.» est plus problématique que "As imperfect as he is, he has been like family to me.». La piste est à explorer ; contentons-nous de suggérer que la relation d'implication notionnelle propre à la concession parait, dans le cas de HOWEVER, moins compatible avec la forme assertive qu'elle ne l'est avec AS. On notera en tout cas, à la suite de Ranger, $(1998$, p. 112), que le schéma avec un seul AS reçoit systématiquement une interprétation concessive en présence de MAY («Wealthy as he may be, ...» = "Although he is...»), là où sans MAY une interprétation causale est également recevable ("Wealthy as he is, ...»= «Although/Since he is...»); en effet, « si l'on introduit une modalisation en MAY, on écarte l'interprétation causale de l'énoncé, car on ne dit rien sur l'existence de $\mathrm{p}$, on ne se prononce que sur sa possibilité d'assertion » (Ranger 1998, p. 117). La même question, de l'incidence de MAY sur l'interprétation, devra être posée pour la structure corrélative en ... AS... AS... ainsi que et surtout pour celle en HOWEVER.

Enfin, qu'est-ce qui distingue l'énoncé d'origine de sa variante en ... MAY... BUT..., qui parait tout à fait recevable ?

And this helps explain, perhaps, my relationship with Reverend Wright. He may be imperfect, but he has been like family to me. He strengthened my faith, ...

En supprimant le double AS, on perd tout renvoi à un gradient sur la notion IMPERFECTION, or ici ce qui importe n'est pas seulement le jeu sur les deux valeurs polaires 'be imperfect' / 'not be imperfect' mais précisément le jeu sur le degré d'imperfection susceptible d'être attribué par autrui au sujet he. Et c'est là qu'intervient MAY, commun aux deux variantes : la prise en compte du co-énonciateur en tant que source possible d'une altérité, dans "As imperfect as he may be (to you, to anyone, even to me ?), (to me) be is like family... » comme dans « He may be imperfect (to you, to anyone, even to me ?), but (to me) ... ». Une fois de plus, le contexte amont « autorise » quiconque, moi y compris, à penser que 'be - be imperfect' est le cas, je n'en maintiens pas moins pour autant que, pour moi, 'he - be like family to me' est, donc reste le cas. L'intérêt, in fine, de cette manipulation, ainsi que de la confrontation de ... MAY... BUT... à l'exemple (3) en WHILE, réside dans le rôle, commun aux deux variantes, du modal MAY.

\section{3. ... MAY...BUT... vs AL/THOUGH}

Nous reviendrons, dans cette dernière partie, sur le schéma corrélatif concessif en ... MAY..., BUT... dont nous sommes partis, pour le comparer plus précisément et en contexte à la subordination en AL/THOUGH, dont le corpus choisi ne comporte, rappelons-le, aucune occurrence. 
Exemple 5: I would not be running for President if I didn't believe with all my heart that this is what the vast majority of Americans want for this country. This union may never be perfect, but generation after generation has shown that it can always be perfected. And today, whenever I find myself feeling doubtful or cynical about this possibility, what gives me the most hope is the next generation - the young people whose attitudes and beliefs and openness to change have already made history in this election.

Quelques mots pour commencer sur le contexte de cet exemple, qui constitue la seconde occurrence, dans ce discours, d'un schéma ... MAY... BUT... qu'on peut incontestablement qualifier de concessif. Le discours même s'intitule, en référence au préambule de la Constitution, " $A$ More Perfect Union », et le propos d'Obama est donc de s'engager à ce que, à l'issue de son mandat présidentiel, "this union is more perfect than it was». Or la valeur 'this union - never be perfect' du domaine associé à la première relation prédicative est présentée comme impliquant «it cannot be perfected» alors que c'est «it can always be perfected» qui est avancé par l'énonciateur ${ }^{16}$; plus précisément, l'opposition porte sur 'never be perfect' / 'never be perfected', la première valeur n'entraînant pas nécessairement la seconde. Toujours est-il que la relation d'entraînement est contestée donc déconstruite, d'où l'interprétation concessive, que confirme la possibilité d'avoir un autre marqueur concessif, modulo des différences sur lesquelles nous allons revenir : "Although this union may never be perfect, (yet) [generation after generation has shown that] it can always be perfected.»

Cet exemple présente un intérêt supplémentaire : le segment qui suit immédiatement à droite, "whenever I find myself doubtful or cynical about this possibility » indique explicitement, avec «doubtful», que le locuteur-énonciateur lui-même n'asserte pas ici, à la différence d'autres exemples du même schéma, la valeur associée à la première proposition, qui reste pour lui du domaine de l'incertain : «Will it ever be perfect, I sometimes find myself feeling doubtful about it. ». Autrement dit, le jeu intersubjectif repose ici sur le dédoublement du locuteur qu'est Obama en énonciateur/coénonciateur, la valeur 'this union - never be perfect' étant autant à mettre au compte d'une altérité foncière ou forte, l'interlocuteur au sens large, qu'à cette altérité interne ou faible que constitue le co-énonciateur. Ce qui confirme, même avec une traduction en français par « peut-être » ("Cette union ne sera peut-être jamais parfaite mais... »), que parler ou non dans le cas du schéma concessif d'un MAY «épistémique » n'a pas de pertinence $^{17}$, la bilatéralité fondamentale de MAY - qui rend d'ailleurs compte de ce que CAN ne lui est jamais substituable dans cet emploi - portant ici non pas sur l'évaluation des chances d'occurrences de la valeur en question selon l'énonciateur dans la situation d'énonciation, mais bien sur le jeu entre l'énonciateur et son autre, doué d'une altérité faible ou forte selon le cas. On notera d'ailleurs qu'au «doubtful»

\footnotetext{
16 On notera que «can always be perfected » dépend de « ... has shown» et que c'est donc preuves à l'appui qu'est avancée cette possibilité clairement unilatérale, d'où CAN.

${ }^{17}$ D'autant que, toujours en français, «certes» est également envisageable : «Cette union ne sera certes jamais parfaite mais...»
} 
répond ensuite "what gives me the most hope », qui reprend le « if I didn't believe with all my heart» rhétorique du début du paragraphe, comme pour revenir et contrebalancer les implications que l'auditoire aurait pu tirer du «doubtful and cynical about this possibility». On est bien ici, une fois de plus et de manière très explicite, dans l'intersubjectif et la rhétorique ; c'est en introduisant «never be perfect» et «doubtful» qu'est le mieux mis en valeur le propos principal, «it can (and will) be perfected» et « gives me hope».

Or, précisément, ce balancement rhétorique si caractéristique d'Obama dans ce discours serait perdu si MAY... BUT était remplacé par ALTHOUGH par exemple (sans autre modification, donc en conservant MAY) :

Exemple 5a: Although this union may never be perfect, [generation after generation has shown that] it can always be perfected.

Le "choix» de (5) plutôt que de (5a) tient selon nous à ce qu'avec (5a), et même avec MAY, la valeur 'this union - never be perfect' est préconstruite comme la valeur attendue, en contradiction possible alors avec la teneur positive du paragraphe qui s'ouvre sur " I wouldn't be running for President if I didn't believe with all my heart that this is what the vast majority of Americans want for this country ». Malgré MAY en effet, cette valeur semble ne pas véritablement pouvoir être remise en question, autrement dit contestée, et l'on perd du coup la dimension interlocutoire, pourtant essentielle en raison de la préconstruction, non seulement de l'inférence déconstruite mais aussi et surtout, par rapport à MAY... BUT, de la valeur 'this union - be perfect' comme prépondérante. On notera en outre qu'ALTHOUGH semble dès lors atténuer l'effet marqué par MAY en bloquant pour ainsi dire la dimension interlocutoire ou intersubjective qu'on lui a associée ailleurs. Nous n'en déduirons pas pour autant que MAY et ALTHOUGH sont redondants ou que MAY «n'ajoute rien»; néanmoins, dans l'expression de la concession, MAY est, cela va de soi, bien moins indispensable avec ALTHOUGH qu'avec BUT, et cela exigerait une étude détaillée d'énoncés en ALTHOUGH avec ou sans MAY que nous ne pouvons mener dans le cadre de cet article ${ }^{18}$.

Nous émettrons, à partir de la comparaison entre (5) et (5a) et en guise de conclusion, l'hypothèse selon laquelle l'absence dans ce discours de la moindre occurrence de THOUGH ou de ses variantes ${ }^{19}$ n'est sans doute pas un hasard. Utiliser THOUGH serait, pour le locuteur, reconnaittre à son autre le droit de penser ce qu'il pense, puisqu'avec ce marqueur la validité de la première relation n'est pas remise en cause (seule l'est l'inférence comme dans tout schéma concessif), mais tout en s'autorisant soi-même à penser autrement. Avec MAY... BUT, en revanche, MAY, en tant que marqueur d'un jeu sur les deux valeurs de la relation prédicative qui lui est associée, entretient un flou, une sorte d'incertitude sur la validation de la relation

\footnotetext{
18 Souesme (2009, p. 170) cite un exemple qui mériterait d'être examiné de près, l'absence de MAY rendant, selon lui et à juste titre, l'énoncé difficile à comprendre : "Although Miss Copley may be a very old friend, Sir Louis is a very influential one and... ».

${ }^{19}$ Parmi les pistes à explorer, en plus de la présence ou non de MAY dans ces subordonnées, se trouve aussi bien entendu celle de la distinction, souvent très subtile, entre THOUGH, ALTHOUGH et EVEN THOUGH entre autres.
} 
prédicative en question, mais une incertitude construite, qui peut donc être feinte et qui, en tout état de cause, ne relève pas de l'extra-linguistique.

De ce point de vue, si THOUGH comme MAY... BUT peuvent l'un et l'autre contribuer à l'expression de la concession, ils ne le font pas de la même façon, le statut de l'altérité étant différent. Avec THOUGH, la valeur associée est préconstruite et l'altérité est de ce fait non contestée ni contestable (exclusion de l'extérieur du domaine, et l'on est en «I et rien d'autre » sans MAY, ou bien non prise en compte de l'extérieur, et l'on est en "I en tout cas » avec MAY), alors qu'avec MAY... BUT l'altérité est prise en compte ('’extérieur n'est pas exclu, bien au contraire, on est alors en «I entre autres » avec I $e t \mathrm{E}$ donc) mais elle est mise au compte d'une instance qui est elle-même dans un rapport d'altérité (faible ou forte) par rapport à l'énonciateur coénonciateur dans un cas, interlocuteur dans l'autre.

\section{Conclusion et prolongements}

Nous reviendrons pour conclure sur nos principales observations et sur les questions laissées en suspens.

La concession est une démarche rhétorique ou pragmatique qui se construit en discours et qui, au-delà de son principe fondateur, la déconstruction d'une relation inférentielle, englobe des cas de figure variés.

Le schéma en ... MAY..., BUT... dont nous sommes partis, est d'une force telle qu'il permet la construction de toute inférence ou presque, même très inattendue ("He may have been to Yale but be's intelligent.» aussi bien que « He may have been to Yale but he's not intelligent. ", « He may be a cop but he isn't a bastard. » tout comme « He may be a cop but he is a bastard.»). Force qui nous parait moindre, mais la démonstration reste à faire, exemples respectifs en contexte à l'appui, avec des opérateurs explicites et exclusifs de la concession tels que ALTHOUGH, THOUGH ou EVEN THOUGH.

La présence de MAY dans le schéma concessif en ... MAY... BUT... lui confère une dimension dialogique ou intersubjective particulièrement explicite, dimension qui, de toute évidence, sert particulièrement bien la cause d'Obama dans ce discours, sans compter que MAY... BUT... se prête davantage que AL/THOUGH à une exploitation ironique de la concession : là aussi, un corpus comparé d'exemples s’impose. Concéder sans céder, autrement dit sauver la face, tel est le dessein plus ou moins conscient d'Obama dans ce discours, réécriture prosaïque de la formule de Charreyre citée en début d'article : «entrer dans le jeu de l'autre sans pour autant considérer que les arguments avancés (...) sont les seuls possibles».

Ce travail ne peut du coup constituer qu'un point de départ d'une étude plus vaste, tant les questions suscitées l'emportent sur les hypothèses esquissées : dans 
quels types de situations ou de discours Obama a-t-il recours à AL/THOUGH ${ }^{20}$, dans quels contextes est-il susceptible d'utiliser en alternance ALTHOUGH et MAY... BUT... par exemple, et en va-t-il autrement pour d'autres orateurs ${ }^{21}$ ? Plus largement, peut-on établir des corrélations entre tel type de situation de discours et tel type de marqueurs de la concession, tant les moyens d'expression de la concession sont variés?

Par ailleurs, et pour en revenir à MAY, quelles conclusions peut-on tirer, à partir d'un corpus d'énoncés avec ou sans MAY dans des conditions de production aussi proches que possible, sur l'incidence de la présence de MAY dans une subordonnée introduite par AL/THOUGH ? Enfin, cela est apparu à plusieurs reprises au cours de ce travail, la comparaison avec le français est d'un intérêt certain (de la traduction ou non de MAY et si oui par quels moyens, à la différence entre les emplois respectifs du IF concessif et du SI concessif).

Telles sont quelques-unes des pistes que ce premier travail sur la variation dans l'expression de la concession nous incite à explorer.

20 Un exemple parmi d'autres, tiré d'un discours prononcé en novembre 2006: «Rather, the question is what strategies, imperfect though they may be, are most likely to achieve the best outcome in Iraq (...).»

21 On pourra consulter Bendinelli, 2011. 


\section{Bibliographie}

BENDINELLI, M., 2011, « Modalité, dialogisme et stratégies discursives : l'auxiliaire modal MAY dans les débats présidentiels américains ", Anglophonia, n³0, p. 163-186.

Charreyre, C., 2005, « Anaphore et identification », L’identification, Cycnos, vol. 21, n¹.

Charreyre C., 2007, «'Du pareil' mais pas 'au même' : EVEN SO, ALL THE SAME, STILL », Les connecteurs, jalons du discours, Peter Lang, p. 157-172.

Chuquet, J. et Oriez, S., 2000, «WHILE : invariance et déformabilité », Cahiers Forell, 14, Université de Poitiers, p. 65-84.

Culioli, A., 1990, Pour une linguistique de l'énonciation. Tome 1. Opérations et représentations. Paris, Ophrys.

Culioli, A., 1999, Pour une linguistique de l'énonciation. Tome 3. Domaine notionnel. Paris, Ophrys.

DE VoGÜÉ, S., 1992, «Aux frontières des domaines notionnels : BIEN QUE, QUOIQUE et ENCORE QUE », L'information grammaticale, 55, p. 23-27.

FluCHA, L., 2005, « Le connecteur AS et l'opération d'identification », L'identification, Cycnos, vol. $21, n^{\circ} 1$.

Huddleston, R. \& Pullum, G. K., 2002, The Cambridge Grammar of the English Language, Cambridge, Cambridge University Press.

Quirk, R., Greenbaum, S., Leech, G. and Svartvik, J., 1985, A Comprehensive Grammar of the English Language, London, Longman.

RANGER, G., 1998, Les constructions concessives en anglais : une approche énonciative, Cahiers de recherche, Numéro spécial, Ophrys.

SEKALI, M., 1992, «Intersubjectivité et implicite par la connexion inter-énoncés : une étude du connecteur anglais BUT », in Perrin, I. (éd.), TELOS n ${ }^{\circ}$ Approches énonciatives de l'énoncé complexe, Louvain-Paris, Peeters, p. 109-119.

SEKALI, M., 2007, "'He's a cop but he isn't a bastard': an enunciative approach to some pragmatic effects of the coordinator BUT», Connectives as Discourse Landmarks, Amsterdam, Benjamins, Pragmatics \& Beyond New Series, 161, p. 155-175.

Souesme, J.-C., 2009, «MAY in concessive contexts », Modality in English: Theory and Description, Salkie, R., Busuttil, P. \& Van Der Auwera, J. (eds), p. 159-176.

Sweetser, E. E., 1990, From Etymology to Pragmatics. Metaphorical and cultural aspects of semantic structure, Cambridge Studies in Linguistics 54, Cambridge, Cambridge University Press. 